\title{
BMJ Open Frailty in the context of rehabilitation interventions for adults: protocol for a scoping review
}

\author{
Kristina M Kokorelias, ${ }^{1,2}$ Sarah Munce, ${ }^{3}$ Kathy S McGilton, ${ }^{2,4}$ Shawna M Cronin, ${ }^{2,5}$ \\ Chen D Wang, ${ }^{1,2}$ Parvin Eftekhar, ${ }^{2,6}$ Darren K Cheng, ${ }^{1,2}$ Shirin Vellani, ${ }^{2,4}$ \\ Susan Jaglal, ${ }^{2,5,6}$ Nancy M Salbach, ${ }^{2,6}$ Pia Kontos, ${ }^{2,7}$ Tracey J F Colella, ${ }^{4,8}$ \\ Alisa Grigorovich, ${ }^{2,7}$ Jill I Cameron ${ }^{2,9}$
}

To cite: Kokorelias KM, Munce S, McGilton KS, et al. Frailty in the context of rehabilitation interventions for adults: protocol for a scoping review. BMJ Open 2019;9:e024838. doi:10.1136/ bmjopen-2018-024838

- Prepublication history and additional material for this paper are available online. To view these files, please visit the journal online (http://dx.doi. org/10.1136/bmjopen-2018024838).

Received 16 June 2018 Revised 5 October 2018 Accepted 16 October 2018

Check for updates

(c) Author(s) (or their employer(s)) 2019. Re-use permitted under CC BY-NC. No commercial re-use. See rights and permissions. Published by BMJ.

For numbered affiliations see end of article.

Correspondence to

Jill I Cameron;

jill.cameron@utoronto.ca

\section{ABSTRACT}

Introduction Although a growing number of frail adults can benefit from rehabilitation services, few are included in rehabilitation services, and reasons for their exclusion are not well understood. To inform research directions in rehabilitation for all adults (aged 18 years and older), we will conduct a scoping review to describe (1) the characteristics of frail adult individuals included in rehabilitation interventions (eg, age range, inclusion and exclusion criteria that are applied), (2) the type of rehabilitation interventions that are used for individuals who are considered frail and (3) the commonly reported outcome measures used for these rehabilitation interventions.

Methods This scoping review will be guided by Arksey and 0'Malley's methodological framework. Ageline, Cochrane CINAHL, Embase, MEDLINE, Pubmed, OTSeeker, PeDRO, PsycINFO and Scopus databases will be systematically searched for articles relevant to rehabilitation interventions and health services. To be eligible for inclusion, studies must report on the outcomes from an intervention that involves all individuals (aged 18 and older) who are considered frail. Only English-language, peer-reviewed publications between 1990 and 2018 will be included. A two-step screening process will consist of (1) a title and abstract review and (2) full-text review. In both levels of screening, a minimum of two investigators will independently screen the title and abstract of all retrieved citations for inclusion against a set of minimum inclusion criteria.

Analysis Results will be presented as a narrative synthesis to facilitate the integration of diverse evidence. Ethics and dissemination This study does not require ethics approval. By examining the current state of rehabilitation interventions for frail adults, this scoping review can offer insight into rehabilitation needs and models of care. It can also guide future rehabilitation research for frail adults. We will share our results with frail adults during a consultation meeting and publish a manuscript in a peer-reviewed rehabilitation journal.

\section{INTRODUCTION}

Rehabilitation healthcare professionals are increasingly met with the challenge of caring for complex and frail patients. Over one

\section{Strengths and limitations of this study}

This review is guided by a well-known methodological framework.

- We will use two independent investigators for all phases of the review.

- This review excludes grey literature and articles published in other languages.

- We will be including only English-language articles published since 1990.

million Canadians are considered medically frail, and this number is expected to reach over two million within the next decade. ${ }^{1}$ Global data suggest that frailty will have an impact worldwide ${ }^{2}$; recent European studies indicate that the prevalence of frailty is $4 \%$ in individuals between 50 and 64 years old and $17 \%$ in individuals aged 65 years or older. ${ }^{3}$ In Canada, the prevalence of frailty in the general population under 65 years of age is estimated at $<10 \%{ }^{4}$

Frailty is a term widely used to signify physical and mental vulnerability. While frailty has been usually defined in the geriatric population, it is increasingly being recognised as being present in younger age groups. ${ }^{5} 6$ Authors broadly define frailty as a common condition that is characterised by deficits in physiological functioning across multiple physical domains. Frailty has further been defined as a physical disability or an impairment in basic or instrumental activities of daily living. ${ }^{7}$ In younger populations, frailty appears to coincide with a chronic or critical condition, which challenges clinicians and researchers to examine this cohort of individuals to establish ways to improve their healthcare trajectories. ${ }^{6}$ Fried et al, using data from the Cardiovascular Health Study, have proposed that a frail individual must display three or more symptoms of weight 
loss, weakness, exhaustion, low activity level and slow gait speed. ${ }^{8}$ Additional research is needed to determine the ideal instruments for identification of frailty in younger patients. ${ }^{5}$

However, the precise definition of frailty among healthcare professionals, administrators and researchers is unclear, presenting challenges for comparisons between studies. ${ }^{4-10}$ Universal criteria for frailty have not been developed $^{11}$ and definitions of frailty, as a syndrome, are undefined in recent publications. ${ }^{4812} 13$

Specifically, there is no clear explication of how frailty is defined in rehabilitation intervention trials, or the impact of frailty in such trials. Moreover, the majority of studies on frailty focus on the older adult population (aged 65 years and older), where frailty is most commonly identified, but exclude adults who are not yet considered 'older' (ie, under the age of 65). By excluding frail younger adults in these studies, rehabilitation services for this population are not well understood and there are no clear directions for how they can be improved on.

The impact of frailty is significant for the individual themselves, but also for their families and the broader healthcare system. ${ }^{4}$ For example, frailty is associated with poor individual outcomes including an increased risk of morbidity and institutionalisation, and places an increased burden on family caregivers (eg, depression and absenteeism). ${ }^{101415}$ Frailty also has multiple clinical and societal consequences as individuals living with frailty are frequent users of primary care, community and residential care, acute care and end-of-life care. ${ }^{189}$

Previous systematic reviews of interventions aimed at reducing frailty have reported on the effectiveness of intervention programmes in frail older adults. ${ }^{11} 16-18$ The majority of the interventions included in these reviews were related to exercise interventions. A systematic review by Theou and colleagues ${ }^{19}$ of exercise interventions to manage frailty found that exercise had a positive impact on all functional outcomes (including mobility, balance and functional performance test batteries).

These reviews identified a variety of definitions of frailty and only used physical capacity or mobility as the measures of frailty. A lack of a universal definition of frailty, as described above, underscores the difficulty in synthesising the intervention literature for frail adults. Moreover, none of these reviews focused on rehabilitation specifically. Rehabilitation approaches to frailty, such as physical therapies, have been associated with improvements in physical, cognitive and social functioning. ${ }^{152021}$ Therefore, the current review, will explore how frailty is operationalised in the context of rehabilitation intervention trials, for adults aged 18 years or older. This review will answer the following research questions: (1) what are the characteristics of frail individuals included in rehabilitation trials (eg, age range, inclusion and exclusion criteria)? (2) What are the types of rehabilitation interventions that are used among individuals who are considered frail? (3) What are the commonly reported outcome measures (related to frailty) for these rehabilitation trials?

\section{METHODS AND ANALYSIS}

We will use the methodological framework proposed by Arksey and $\mathrm{O}^{\prime} \mathrm{Malley}^{22}$ to guide this scoping review. A scoping review is used to profile the existing research on a topic and identify areas of future study. ${ }^{23}$ Given the breadth of the current research questions, this approach was deemed appropriate.

This framework has six stages: (1) identify the research question; (2) identify relevant studies; (3) select studies; (4) chart the data; (5) collate, summarise and report the results and (6) consult with key stakeholders, such as clinical members of the research team. ${ }^{22}$ Although typically applied to systematic reviews, the Preferred Reporting Items for Systematic Reviews and Meta-Analyses for Protocols (PRISMA-P) ${ }^{24}$ was used to prepare this scoping review protocol. Consistent with the aims of a scoping review, we will not access the quality of the literature or synthesise quantitative findings. ${ }^{23}$

\section{Eligibility criteria}

We are limiting our search to experimental and quasi-experimental designs, as these are typically regarded as the highest level of evidence according to the Canadian Task Force on the Periodic Health Examination. ${ }^{25}$ We are conceptualising a rehabilitation trial as an experiment or test of the quality, usefulness or benefit of 'evaluative, diagnostic and therapeutic interventions whose purpose is to restore functional ability or enhance residual functional capability' in adults. ${ }^{2}$ Rehabilitation trials may or may not include typical rehabilitation interventions such those used in physical therapy, occupational therapy, speech-language pathology and rehabilitation psychology. ${ }^{26}$ For the purpose of this review, we will adopt the definition of frailty proposed by the Canadian Frailty Network (CFN): 'Frailty is a state of increased vulnerability, with reduced physical reserve and loss of function across multiple body systems. This reduces the ability to cope with normal or minor stresses, which can cause rapid and dramatic changes in health'. ${ }^{1}$ The CFN is a Canada-wide initiative aimed at improving the care for Canadians living with frailty through the increased recognition of frailty. ${ }^{1}$

For the purpose of this review, articles will be included if:

1. Authors make reference to frailty in the context of their inclusion/exclusion criteria, sample population, intervention or outcome measures.

2. They present findings from an evaluation of a rehabilitation intervention. Rehabilitation interventions are defined as 'evaluative, diagnostic and therapeutic interventions whose purpose is to restore functional ability or enhance residual functional capability' in adults. $^{27}$ 
3. They include experimental or quasi-experimental study designs. We define quasi-experimental as an experimental study design without random assignment.

4. They are peer reviewed and published in English. Only studies published from January 1990-date of submission will be included. We have identified 1990 as a time period where frailty began being conceptualised in the rehabilitation literature.

Studies will be excluded if they do not have an a priori definition of frailty.

\section{Search strategy}

Searches will be conducted in Ageline, Cochrane Central Register of Controlled Trials (CENTRAL), CINAHL, Cochrane Database of Systematic Reviews, Embase, Ovid MEDLINE (R) (including Epub Ahead of Print, In-Process and Other Non-Indexed Citations, Ovid MEDLINE(R) Daily), Pubmed, OTSeeker, PeDRO, PsycINFO and Scopus. Search strategies will include the use of text words and subject headings (eg, MeSH and Emtree) related to (1) frailty and (2) rehabilitation (see online supplementary material 1 for an example of MEDLINE search terms). The search strategy will be peer reviewed using Peer Review of Electronic Search Strategies guidelines. ${ }^{28}$

\section{Study selection}

The review process will consist of two levels of screening: (1) a title and abstract review and (2) full-text review. In both levels of screening, two investigators will independently screen the title and abstract of all retrieved citations for inclusion against the set of predefined inclusion criteria. If discrepancies are identified, a third reviewer will review the relevant articles and a discussion with all reviewers will occur until consensus is reached. Research team members will contribute to the selection of articles, data abstraction, data synthesis and preparation of manuscripts for publication.

\section{Data abstraction and collection}

A standardised, piloted data extraction form created by the authors will be used when extracting data from studies meeting inclusion criteria. To ensure inter-rater reliability, the data abstraction form will be pilot tested with a subset of the articles and modified to enhance extractor agreement and fit with information presented in included articles.

Abstracted data will include study characteristics (eg, year of publication and country of study), the definition of frailty (how the authors conceptualise or define frailty, if included), sample size, participant population (eg, chronic disease condition, percent considered frail, characteristics of frail population, income, ethnicity, age, sex, education, inclusion and exclusion criteria), characteristics of rehabilitation intervention (focus, duration, frequency, delivery setting, mode of delivery), outcome (measures) collected and results (ie, efficacy or effectiveness of the intervention), study design (eg, randomised control trial) and the relationship between frailty and study outcomes (ie, how did frailty influence study outcomes).

\section{Quality assessment}

Study quality will not be evaluated as the purpose of a scoping review is to identify gaps in the literature and future areas for a systematic review. ${ }^{29}$ No study will be excluded based on the quality assessment. Covidence software will be used to manage the records and data throughout the review. ${ }^{30}$ A PRISMA diagram as developed and maintained by Covidence will be exported and included in the results. ${ }^{26}$

\section{Synthesis}

Consistent with the purpose of scoping reviews, we will provide a summary and synthesis of the results. We will follow Levac and colleagues ${ }^{29}$ and break this into three distinct steps: (1) analysis, (2) reporting the results, as they relate to our objective and research questions and (3) relate the findings to the implications they may have for future rehabilitation research, clinical practice and health service policy.

\section{Consultation}

We aim to share our preliminary findings with the CFN through their monthly webinars. We will also be sharing our preliminary findings and results locally at monthly meetings at Toronto Rehabilitation Institute-University Health Network. These meetings are attended by primary care providers, nurses, occupational therapists, physiotherapists, economists and rehabilitation and social scientists not associated with the research team. We anticipate at least 5 to 15 participants at these meetings. In all meetings, we will take detailed notes of the discussion that occurs. We will summarise their feedback and include it in our qualitative thematic analysis and results. We will also use these meetings to discuss the discuss potential implications that findings may have on future research, clinical practice and policy. We will not be using a predetermined framework to guide their feedback.

\section{Patient and public involvement statement}

Patients and public are not involved in this research.

\section{ETHICS AND DISSEMINATION}

This scoping review will guide future research aimed at supporting rehabilitation for frail adults by highlighting which individuals are (and are not) included in experimental and quasi-experimental studies, and providing insight into their rehabilitation needs and models of care. Depending on the findings, there is potential for a subanalysis of the results with respect to age range and health status/condition that may inform different types of rehabilitation interventions and models for individuals who are considered frail. This knowledge will offer insight into how generalisable findings from rehabilitation interventions are to all frail adults, and what gaps in current rehabilitation interventions for frail adults 
exist. In addition to our monthly meetings, we will disseminate our findings through traditional knowledge translation mechanisms, including publication in peer-reviewed journals and conference proceedings. This will ensure that our findings are available to the appropriate academic and clinical audiences. We will continue to seek out partnerships with local, provincial and/or national research and health initiatives so that the results are disseminated in a timely and effective manner (eg, at stakeholder meetings, discussions with the local health networks). To disseminate our findings with individuals who are considered frail awe aim to use partnerships with appropriate health initiatives (eg, frailty organisations and health clinics were frail individuals frequent) to distribute lay summaries of our findings via the CFN.

We acknowledge some limitations of this review. This review will not include grey literature, limiting the search to rehabilitation interventions that include frail adults but are not peer reviewed. This review will be limited to English-language studies which may result in a bias in results towards predominantly English-language speaking countries.

The currently proposed scoping review has a number of strengths. First, this review is guided by a well-known methodological framework and all phases will be conducted in duplicate. Moreover, this review is being guided by the PRISMA-P, ensuring systematic and rigorous methods are in place. ${ }^{24}$ This review will contribute to critical and emerging perspectives on frailty within rehabilitation. The results of the review will also advance our knowledge on the generalisability of the existing evidence on rehabilitation interventions for frail adults and delineate any promising preventive and therapeutic actions for adults who are considered frail. Moreover, this review will comment on the ethical implications (eg, lack of programmes for persons with dementia) that exist, should our review show a lack of literature that addresses the benefits of rehabilitation in frail patients. Often 'not studied' is conflated with 'no evidence' which leads to withdrawal of services targeted at vulnerable populations. This scoping review will identify gaps in rehabilitation services that need to be addressed for adults who are considered frail.

\section{Author affiliations}

${ }^{1}$ Rehabiliation Sciences Institute, University of Toronto, Toronto, Ontario, Canada ${ }^{2}$ Toronto Rehabilitation Institute - University Health Network, Toronto, Ontario, Canada

${ }^{3}$ LIFEspan Service, Toronto Rehabilitation Institute, Toronto, Ontario, Canada ${ }^{4}$ Faculty of Nursing, University of Toronto, Toronto, Ontario, Canada ${ }^{5}$ Institute of Health Policy Management and Evaluation, University of Toronto, Toronto, Ontario, Canada

${ }^{6}$ Department of Physical Therapy, University of Toronto, Toronto, Ontario, Canada ${ }^{7}$ Dalla Lana School of Public Health, University of Toronto, Toronto, Ontario, Canada ${ }^{8}$ Toronto Rehabilitation Institution, University Health Network, Cardiac Rehabilitation and Prevention Program, Toronto, Ontario, Canada

${ }^{9}$ Department of Occupational Science and Occupational Therapy, University of Toronto, Toronto, Ontario, Canada
Contributors Allauthors contributed to the project idea. KSM and SJ initiated the project. KMKwrote the first draft of this protocol and revised the article during thereview process. She provided guidance to the Information Specialist withrespect to the design of the search strategy. KMK and JIC finalized theliterature search strategy, with feedback from all authors. KMK, SMC, CDW, PE,DKC, SV and JIC piloted the search strategy. SM provided methodologicalguidance on the development of this protocol and reviewed the original and revised manuscript. NMSprovided contextual guidance on rehabilitation trials and interventions. JIC, SMC,CDW, DKC, SV, KSM, PK, TJFC and AG were involved in editing and revising theprotocol. All authors approved the final version of the protocol and areaccountable for all aspects of the work.

Funding The authors have not declared a specific grant for this research from any funding agency in the public, commercial or not-for-profit sectors.

Competing interests None declared.

Patient consent for publication Not required.

Provenance and peer review Not commissioned; externally peer reviewed.

Open access This is an open access article distributed in accordance with the Creative Commons Attribution Non Commercial (CC BY-NC 4.0) license, which permits others to distribute, remix, adapt, build upon this work non-commercially, and license their derivative works on different terms, provided the original work is properly cited, appropriate credit is given, any changes made indicated, and the use is non-commercial. See: http://creativecommons.org/licenses/by-nc/4.0/.

\section{REFERENCES}

1. Canadian Frailty Network 2018. Frailty in Canada. http://www.cfnnce.ca/frailty-in-canada/ (Accessed 21 Feb 2018).

2. Romero-Ortuno $\mathrm{R}$, Walsh $\mathrm{CD}$, Lawlor $\mathrm{BA}$, et al. A frailty instrument for primary care: findings from the Survey of Health, Ageing and Retirement in Europe (SHARE). BMC Geriatr 2010;10:57.

3. Santos-Eggimann B, Cuénoud P, Spagnoli J, et al. Prevalence of frailty in middle-aged and older community-dwelling Europeans living in 10 countries. J Gerontol A Biol Sci Med Sci 2009;64:675-81.

4. Rockwood K, Song X, Mitnitski A. Changes in relative fitness and frailty across the adult lifespan: evidence from the Canadian National Population Health Survey. CMAJ 2011;183:E487-94.

5. Bagshaw M, Majumdar SR, Rolfson DB, et al. A prospective multicenter cohort study of frailty in younger critically ill patients. Crit Care 2016;20:175.

6. Bonner S, Lone NI. The younger frail critically ill patient: a newly recognised phenomenon in intensive care? Crit Care 2016;20:349.

7. Hogan DB, MacKnight $\mathrm{C}$, Bergman $\mathrm{H}$. Models, definitions, and criteria of frailty. Aging Clin Exp Res 2003;15:1-29.

8. Fried LP, Ferrucci L, Darer J, et al. Untangling the concepts of disability, frailty, and comorbidity: implications for improved targeting and care. The Journals of Gerontology Series A: Biological Sciences and Medical Sciences, 2004:M255-63.

9. Buckinx F, Rolland Y, Reginster JY, et al. Burden of frailty in the elderly population: perspectives for a public health challenge. Arch Public Health 2015;73:19.

10. Torpy JM, Lynm C, Glass RM. Frailty in older adults. JAMA 2006;296:2280.

11. Arantes P, Alencar M, Dias R, et al. Physical therapy treatment on frailty syndrome: systematic review. Rev Bras Fisioter 2009;13:365-75.

12. Rockwood K. Conceptual models of frailty: accumulation of deficits. Can J Cardiol 2016;32:1046-50.

13. Mitnitski AB, Graham JE, Mogilner AJ, et al. Frailty, fitness and latelife mortality in relation to chronological and biological age. BMC Geriatr 2002;2:1.

14. Strandberg TE, Pitkälä KH, Tilvis RS. Frailty in older people. Eur Geriatr Med 2011;2:344-55.

15. Cameron ID, Fairhall N, Gill L, et al. Developing Interventions for Frailty. Adv Geriatr 2015;2015:1-7.

16. de Labra C, Guimaraes-Pinheiro C, Maseda A, et al. Effects of physical exercise interventions in frail older adults: a systematic review of randomized controlled trials. BMC Geriatr 2015;15:154.

17. Giné-Garriga $M$, Roqué-Fíguls $M$, Coll-Planas $L$, et al. Physical exercise interventions for improving performance-based measures of physical function in community-dwelling, frail older adults: a systematic review and meta-analysis. Arch Phys Med Rehabil 2014;95:753-69.

18. Chin A Paw MJ, van Uffelen JG, Riphagen I, et al. The functional effects of physical exercise training in frail older people : a systematic review. Sports Med 2008;38:781-93. 
19. Theou O, Stathokostas L, Roland KP, et al. The effectiveness of exercise interventions for the management of frailty: a systematic review. J Aging Res 2011;2011:1-19.

20. Quittan M. [Frailty from the rehabilitation medicine point of view]. Z Gerontol Geriatr 2014;47:385-8

21. Puts MTE, Toubasi S, Andrew MK, et al. Interventions to prevent or reduce the level of frailty in community-dwelling older adults: a scoping review of the literature and international policies. Age Ageing 2017;46:383-92.

22. Arksey H, O'Malley L. Scoping studies: towards a methodological framework. Int J Soc Res Methodol 2005;8:19-32.

23. Gagliardi AR, Berta W, Kothari A, et al. Integrated knowledge translation (IKT) in health care: a scoping review. Implementation Science 2015;11:38.

24. Moher D, Shamseer L, Clarke M, et al. Preferred reporting items for systematic review and meta-analysis protocols (PRISMA-P) 2015 statement. Syst Rev 2015;4:1
25. Burns PB, Rohrich RJ, Chung KC. The levels of evidence and their role in evidence-based medicine. Plast Reconstr Surg 2011;128:305-10.

26. Boston Working Group. Boston working group on improving health care outcomes through geriatric rehabilitation. Med Care 1997;35:JS4-JS20.

27. Wade DT. Outcome measures for clinical rehabilitation trials: impairment, function, quality of life, or value? Am J Phys Med Rehabil 2003;82:S26-31.

28. McGowan J, Sampson M, Salzwedel DM, et al. PRESS Peer Review of Electronic Search Strategies: 2015 Guideline Statement. J Clin Epidemiol 2016;75:40-6.

29. Levac D, Colquhoun H, O'Brien KK. Scoping studies: advancing the methodology. Implement Sci 2010;5:69.

30. Veritas Health Innovation. Covidence systematic review software. Melbourne Australia: Veritas Health Innovation. 